\title{
Microalgas como alternativa sostenible para la producción de biodiesel
}

Fecha de recepción: 29 de julio de 2019

Fecha de aprobación: 18 de noviembre de 2019

\author{
Isabel Cristina Castellanos ${ }^{1}$ \\ Universidad Ean \\ iccastellan@universidadean.edu.co \\ Kenia González-Peralta ${ }^{2}$ \\ Universidad Ean \\ kgonzal59724@universidadean.edu.co \\ Santiago Jose Pinzón-Torres \\ Universidad Ean \\ spinzon19526@universidadean.edu.co
}

DOI: https://doi.org/10.21158/23823399.v6.n0.2018.2425

Cómo citar este artículo: Castellanos, I. C.; González-Peralta, K.; Pinzón-Torres, S. J. (2018). Microalgas como alternativa sostenible para la producción de biodiesel. Revista Ontare. 6, (83-109). DOI: https://doi.org/10.21158/23823399.v6.n0.2018.2425

\section{Resumen}

Los recientes problemas ambientales asociados a la contaminación atmosférica evocan un especial énfasis investigativo en la búsqueda de fuentes de energía que remplacen los combustibles fósiles, uno de los mayores generadores de contaminación. Desde hace varios años el biodiesel se ha convertido en una alternativa para esta problemática. Este biocombustible se puede producir a partir de diferentes materias primas que minimizan la generación de contaminantes, no obstante, el uso de aceites vegetales o grasas animales como materia prima origina problemáticas asociadas a la deforestación con relación al monocultivo y la promoción de actividades ganaderas.

1 Magíster en Ciencias Bioquímica de la Universidad de Colombia. Pregrado en Química, la Universidad de Colombia. ORCID: https://orcid.org/0000-0001-6299-8505

2 Estudiante del programa de Ingeniería Química de la Universidad Ean. ORCID: https://orcid. org/0000-0001-7671-186X

3 Estudiante del programa de Ingeniería Ambientalde la Universidad Ean. ORCID: https://orcid. org/0000-0001-7113-643X 
Bajo este panorama, la producción de biodiesel con base en microalgas como fuente de lípidos se ha proyectado como una alternativa sostenible para la industria de biocombustibles. Esta revisión presenta la producción de lípidos a partir de microalgas y plantea nuevos retos dentro de la industria energética, ya que las microalgas se postulan como una fuente de biodiesel renovable que es capaz de satisfacer la demanda mundial de combustibles para el transporte, sin poner en riesgo aspectos sociales ni ambientales. Las microalgas, además de ser la materia prima para el biodiesel, brindan una alternativa en la descontaminación del ambiente, especialmente del aire y del agua. Su producción es económicamente viable, equitativa dentro de la sociedad y ambientalmente soportable; en una palabra, es sostenible.

\section{Palabras clave}

Biocombustibles; biodisel; producción de biodiesel; producción de biocombustibles; industria energética; nuevas fuentes de energía; energías limpias; sostenibilidad. 


\title{
Microalgae as a sustainable alternative for biodiesel production
}

\begin{abstract}
The recent environmental problems that are associated with air pollution evoke a special research emphasis on the search for sources of energy that replace fossil fuels, one of the biggest pollution generators. For several years, biodiesel has become an alternative for this problem. This biofuel can be produced from different raw materials that minimize the generation of pollutants, however, the use of vegetable oils or animal fats as raw material bring on problems associated with deforestation in relation to monoculture and the promotion of stockbreeding activities.

Under this scenario, the production of biodiesel based on microalgae as a source of lipids has been projected as a sustainable alternative for the biofuel industry. This review presents the production of lipids from microalgae, and poses new challenges within the energy industry, since microalgae are postulated as a renewable biodiesel source that is able to meet the global demand for transport fuels, without putting at risk social or environmental aspects. In addition to being the raw material for biodiesel, microalgae provide an alternative in the decontamination of the environment, especially the air and water. Its production is economically viable, equitable within society, and environmentally bearable; in a word, it is sustainable.
\end{abstract}

\section{Keywords:}

Biofuels; biodiesel; biodiesel production; biofuel production; energetic industry; new sources of energy; clean energies; sustainability. 


\title{
Microalgas como alternativa sustentável para produção de biodiesel
}

\begin{abstract}
Resumo
Os recentes problemas ambientais associados à poluição do ar evocam uma ênfase especial de pesquisa na busca de fontes de energia que substituam os combustiveis fósseis, um dos maiores geradores de poluição. Por vários anos, o biodiesel tornouse uma alternativa para esse problema. Esse biocombustivel pode ser produzido a partir de diferentes matérias-primas que minimizam a geração de poluentes, no entanto, o uso de óleos vegetais ou gorduras animais como matéria-prima causa problemas associados ao desmatamento em relação à monocultura e à promoção de atividades pecuárias.

Nesse cenário, a produção de biodiesel à base de microalgas como fonte de lipídios foi projetada como uma alternativa sustentável para a indústria de biocombustíveis. Esta revisão apresenta a produção de lipídios a partir de microalgas e coloca novos desafios no setor de energia, uma vez que as microalgas são postuladas como uma fonte renovável de biodiesel capaz de atender à demanda global por combustíveis de transporte, sem colocar aspectos sociais ou ambientais de risco.

Além de ser a matéria-prima do biodiesel, as microalgas oferecem uma alternativa na descontaminação do meio ambiente, principalmente ar e água. Sua produção é economicamente viável, equitativa na sociedade e ambientalmente suportável; em resumo é sustentável.
\end{abstract}

\section{Palavras-chave}

Biocombustíveis; biodiesel; produção de biodiesel; produção de biocombustível; setor de energia; novas fontes de energia; energias limpas; sustentabilidade. 


\title{
Les microalgues comme alternative durable pour production de biodiesel
}

\begin{abstract}
Résumé
Les récentes problématiques environnementales liées à la pollution de l'air montrent un regain d'intérêt particulier pour la recherche de sources d'énergie alternatives remplaçant les combustibles fossiles considérés comme les principaux responsables de la pollution atmosphérique. Le biodiesel est ainsi devenu l'une des solutions privilégiées pour faire face à cette problématique dans la mesure où ce biocarburant est produit à partir de différentes matières premières végétales et minimise la génération de gaz à effet de serre. Cependant, l'utilisation de matières premières d'origine végétale ou animale pose des problèmes environnementaux liés à la déforestation, à la monoculture et à la prolifération de l'élevage.

$C$ 'est dans ce contexte que la production de biodiesel de microalgues peut devenir une alternative durable pour l'industrie des biocarburants. Cet article analyse également la production d'huile de microalgues et révèle les nouveaux défis du secteur énergétique dans la mesure où cette matière première végétale est une source d'énergie renouvelable capable de répondre à la demande mondiale de carburants, sans risques environnementaux ni sociaux.
\end{abstract}

Outre le fait d'être le composant essentiel du biodiesel, les microalgues constituent une alternative à la décontamination de l'environnement, principalement de l'air et de l'eau, grâce à une production économiquement viable, équitable au sein de la société et écoresponsable. En un mot, c'est l'un des carburants du futur.

\section{Mots-clés}

Biocarburants; biodiesel; production de biodiesel; production de biocarburants; industrie de l'énergie; nouvelles sources d'énergie; énergies propres; durabilité. 


\section{Introducción}

L

os problemas de contaminación atmosférica ocasionados por la producción de combustibles fósiles convocan a la academia y a los sectores industriales a enfocar esfuerzos en el planteamiento y la implementación de nuevas alternativas sostenibles en el propósito de reemplazar los combustibles fósiles. El biodiesel, hoy, se ha convertido en la propuesta para mitigar estas problemáticas, de modo que adquiere un rol importante frente a la necesidad de buscar energías limpias que disminuyan la concentración de óxidos de azufre, hidrocarburos aromáticos y demás subproductos de la combustión incompleta en el ambiente que provienen de la combustión del diesel de origen fósil, en busca de soluciones orientadas a reducir el efecto de la lluvia ácida y la contaminación ambiental (Basha, Gopal y Jebaraj, 2009).

En los últimos años, los biocombustibles han ingresado al mercado apoyados por la adopción de decretos de ley que permiten la introducción de estos combustibles alternativos. En Colombia se aprobó la obligatoriedad de la mezcla de biocombustibles desde el 2004 (Ley 939 del 2004), con porcentajes de inclusión que iniciaron en el $7 \%$ y han incrementado de manera progresiva hasta el $12 \%$ que registran en la actualidad. A partir del 25 de febrero del 2019, el porcentaje se estableció en el $12 \%$ de inclusión de biodiesel (Resolución 40174 de 2019). Esta medida se mantuvo hasta mayo de este año y se retomará de nuevo en agosto, con el objeto de llegar en nuestro país a una meta final en el 2020 del $20 \%$.

El biodiesel es biodegradable, no deja residuos tóxicos ni peligrosos y contiene oxígeno en su estructura química. Gracias a esto se promueve la combustión completa, lo que reduce las emisiones de monóxido de carbono o CO en los motores (Zhu, Cheung, Zhang y Huang, 2011). Estos beneficios llevan a que el biodiesel que se produce bajo los estándares 
óptimos -ASTM D 6751 — tome un papel importante como energía limpia, pues no genera contaminantes ni residuos.

La fabricación del biodiesel se puede llevar a cabo desde una gran variedad de materias primas, entre las cuales las más comunes son los aceites vegetales. No obstante, al usar estos aceites como material de partida en la producción básica surgen nuevas problemáticas. Por una parte, la competencia económica en el mercado por el uso del aceite dentro de la canasta familiar afectará inevitablemente la seguridad alimentaria. Así, se proyecta detrás de este nuevo recurso un problema de orden social, además del conocido problema ambiental por el fomento del monocultivo de palma de aceite (Gao, Skutsch, Masera y Pacheco, 2011). Dadas estas dificultades que hacen de la producción de biocombustibles hoy un proyecto no soportable para el medio ambiente ni equitativo dentro de la sociedad, la búsqueda y la puesta en marcha de alternativas sostenibles para la producción de los lípidos en la producción del biodiesel es una necesidad.

Es evidente que la producción actual de biodiesel desde aceites vegetales no cumple con las expectativas de sostenibilidad: la deforestación y los cambios de uso en algunos suelos forestales son algunas de las razones ambientales. Según Fedepalma (López, 2018), el área sembrada en Colombia para la producción de aceite de palma pasó de 5000 ha en 1962 a superar hoy las 500000 ha bajo la promesa de la reducción de emisiones asociadas a un menor uso de combustibles fósiles. Sin embargo, es importante cuestionar si esta promesa frente a perder hectáreas de bosque tropical que consumen toneladas de $\mathrm{CO} 2$ vale la pena.

Contrario a este panorama, las algas oleaginosas hoy se consideran fuente de desarrollo energético y un recurso renovable (Johnson y Wen, 2009; Loera-Quezada y Olguín, 2010; Mandal y Mallick, 2009). Son organismos exitosos tanto evolutiva como ecológicamente. Crecen de manera rápida debido a su estructura simple y logran adaptarse con facilidad a 
diferentes condiciones de estrés ambiental. Su producción a gran escala tiene la capacidad de generar biomasa rica en lípidos de forma rápida y eficiente (Monari, Righi y Olsen, 2016) con menores requimientos de superficie, en comparación con los cultivos agrícolas y sin necesidad de pesticidas. Sumado a estas ventajas, las microalgas, un grupo diversos de microorganismos fotosintéticos procarióticos y eucariotas, poseen la capacidad de absorber $\mathrm{CO}_{2}$, con lo cual mitigan la huella de carbono, y así de producir lípidos, por lo que se consideran con potencial para la obtención de biocombustibles de tercera generación.

Así mismo, las microalgas, además de ser una excelente fuente de materia prima para biodiesel, también son una alternativa que permite mitigar la contaminación del aire y del agua. Del aire, como se mencionó, en razón a que parten de $\mathrm{CO}_{2}$ atmosférico para realizar procesos fotosínteticos. Del agua, dada su capacidad fitorremediadora en la remoción de nitrógeno y fósforo, según estudios que lo han demostrado en aguas residuales (Bermeo Castillo, 2011; Bohutskyi et al., 2015; Clebot, 2018; Monari, Righi y Olsen, 2016).

A fin de realizar un cultivo de microalgas existen tres metodologías con relación al sistema empleado (Milano et al., 2016): abierto, cerrado e híbrido. El sistema abierto consiste en desarrollar el cultivo de microalgas en piscinas al aire libre, por lo que las microalgas absorben los nutrientes de su entorno. El sistema cerrado consiste en tener el cultivo de microalgas en recipientes totalmente cerrados, conocidos como «fotobiorreactores» (Hernández-Pérez y Labbé, 2014; Merchuk, Gluz y Mukmenev, 2000; Yeh y Chang, 2011), en los que todos los nutrientes necesarios para la supervivencia y la reproducción de las microalgas los suministra el cultivador; finalmente, el sistema híbrido, el cual consiste en cultivar primero las microalgas en un sistema cerrado y después pasarlas a un sistema abierto con el objetivo de que absorban los nutrientes del entorno. 
En el proceso de cosecha es necesario separar el agua y dejar una pasta húmeda de microalgas (Christenson y Sims, 2011), luego, el proceso se divide en dos pasos. El primero consiste en acumular las microalgas y, posteriormente, separar la biomasa mediante métodos, como, por ejemplo, la floculación con la utilización de agentes floculantes, es decir, $\mathrm{FeCl}_{3}, \mathrm{Fe}_{2}(\mathrm{SO} 4)_{3}$ ó $\mathrm{Al}_{2}(\mathrm{SO} 4)_{3}$ entre los más comunes. Despues de obtener la biomasa se procede al segundo paso que consiste en concentrar la biomasa mediante técnicas tales como la centrifugación y la filtración (Milano et al., 2016). Después de la cosecha viene la etapa en la que ocurre la extracción de lípidos de la biomasa. En esta etapa se debe someter la biomasa obtenida a un proceso de secado y, posteriormente, una vez la biomasa se encuentra seca, se realiza la extracción de los lípidos con procesos físicos, químicos - por medio de solventes orgánicos-o métodos en los que se utilizan fluidos supercríticos (Milano et al., 2016). Con los lípidos obtenidos inicia el proceso para la producción del biocombustible. La producción se realiza por medio de la transesterificación de los triglicéridos, aunque también es posible realizarla en un proceso bioquímico denominado «interesterificación», el cual consiste en un reordenamiento al azar o dirigido de los grupos acilo dentro de un mismo triacilglicerol (Sreenivasan, 1978); sin embargo, este procedimiento es más costoso.

\section{Microalgas como materia prima para la producción de biodiesel}

\footnotetext{
T as microalgas, dado su gran potencial para la produccion de lípidos, se han convertido en una excelente alternativa en la fabricación de biodiesel. Las microalgas convierten $\mathrm{CO}_{2}$ y nutrientes del agua en biomasa (Amaro, Guedes y Malcata, 2011). Otros factores potenciadores de las microalgas como microreactores generadores de materia prima para la fabricación del biodiesel son la alta tasa de crecimiento poblacional y la facilidad que presentan en su cultivo, ya que las microalgas tienen
} 
requerimientos nutricionales sencillos que favorecen su crecimiento en casi cualquier ambiente.

Así mismo, las microalgas cuentan con un factor diferenciador con respecto al resto de las materias primas comunes, esto es, los aceites vegetales y las grasas animales. En este sentido, las microalgas no generan una competencia económica con la canasta familiar, lo que las postula como una solución a diferentes a problemas ambientales actuales. Poseen capacidades metabólicas que permiten abordar de forma simultánea más de un objetivo: de una parte, absorben grandes cantidades de $\mathrm{CO}_{2}$, lo que es una alternativa en el propósito de reducir el efecto invernadero, $y$, de otra, se ha promovido su uso sobre grandes cuerpos de agua contaminados para la reducción de la formación de lodos, la disminución de la población bacteriana nociva para el ambiente y la eliminación o biotransformación de contaminantes, lo que incluye nutrientes - nitrógeno y fósforo - y xenobióticos (Tripathi, Gupta y Thakur, 2019), en razón a su capacidad de realizar ficorremediación.

Para la fabricación de biodiesel mediante microalgas como materia prima se realizan los cinco pasos que se esquematizan en la figura 1. 
Figura 1. Mapa conceptual de producción de biodiesel a partir de microalgas

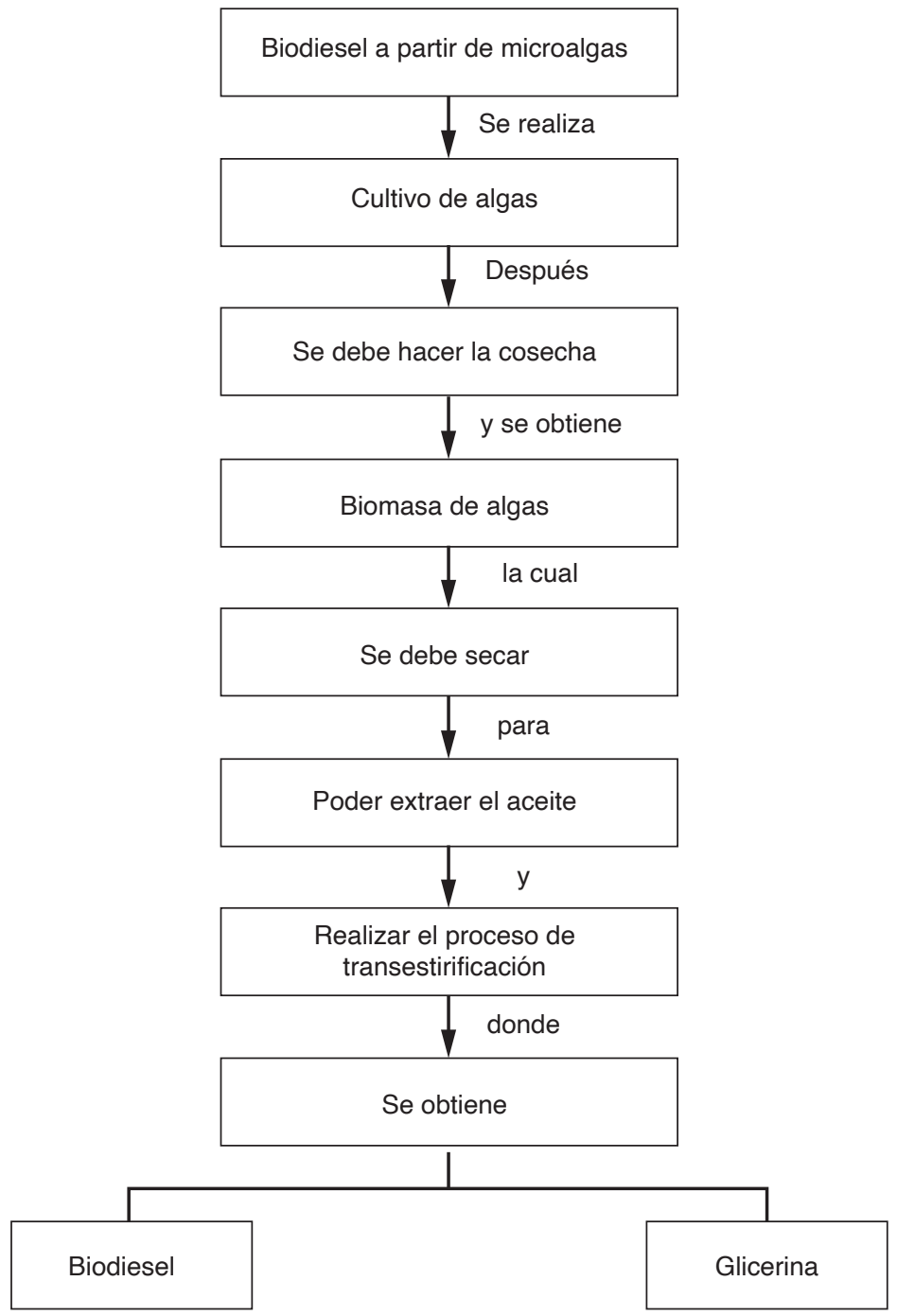

Fuente. Elaboración propia con el software Draw.io. 


\section{Especies de microalgas para la producción de biodiesel}

11 contenido de lípidos de las microalgas depende de su especie y de las condiciones de cultivo (Milano et al., 2016), aunque el con-
tenido de lípidos puede variar entre un $5 \%$ y un $70 \%$ en peso de masa seca, dependiendo de condiciones como, por ejemplo, el estrés, ya que las microalgas tienen capacidad para sobrevivir en condiciones diversas y extremas que se reflejan, muchas veces, en un inusual patrón de lípidos celulares que puede llegar a aumentar hasta en un $90 \%$ en ambientes estresados. En estos se realiza un control biosintético de la composición química por estrés nutricional por deficiencia de nitrógeno, fósforo, azufre y silicio o estrés ambiental por cambios ambientales tales como la salinidad y el $\mathrm{pH}$ del medio de cultivo, la temperatura y la intensidad luminosa. La deficiencia de nitrógeno es, con respecto a los nutrientes, el factor que más afecta el metabolismo de los lípidos (Arunachalam, Sivanandi, Pandian, Arumugamurthi y Sircar, 2018; Shuba y Kifle, 2018).

Las microalgas también contienen proteínas (Becker, 2007) y carbohidratos que se extraen para la produción de etanol, otro biocombustible (Chen et al., 2013). En la tabla 1 se muestran algunas de las especies de microalgas que mayor número de estudios reportan junto con los porcentajes en materia seca de lípidos, proteínas y carbohidratos; además, estas microalgas tienen la capacidad de crecer de manera rápida y acumular largas cadenas de lípidos naturales en el interior de sus células. 
Tabla 1. Especies representativas de microalgas y sus porcentajes en materia seca de lípidos, proteínas y carbohidratos

\begin{tabular}{|c|c|c|c|}
\hline Microalgas & Lípidos (\%) & Proteína (\%) & Carbohidratos (\%) \\
\hline Anabaena cylindrica & $4-7$ & $43-56$ & $25-30$ \\
\hline Aphanizomenon flos-aquae & 3 & 62 & 23 \\
\hline Arthrospira maxima & $6-7$ & $60-71$ & $13-16$ \\
\hline Botryococcus braunii & 86 & 4 & 20 \\
\hline Chlamydomonas rheinhardii & 21 & 48 & 17 \\
\hline Chlorella ellipsoidea & 84 & 5 & 16 \\
\hline Chlorella pyrenoidosa & 2 & 57 & 26 \\
\hline Chlorella sorokiniana & $20-36$ & $21-53$ & $5-25$ \\
\hline Chlorella vulgaris & $14-22$ & $51-58$ & $12-17$ \\
\hline Dunaliella salina & 6 & 57 & 32 \\
\hline Dunaliella bioculata & 8 & 49 & 4 \\
\hline Euglena gracilis & $14-20$ & $39-61$ & $14-18$ \\
\hline Prymnesium parvum & $22-38$ & $30-45$ & $25-33$ \\
\hline Porphyridium cruentum & $9-14$ & $28-39$ & $40-57$ \\
\hline Scenedesmus obliquus & $12-14$ & $50-56$ & $10-17$ \\
\hline Scenedesmus quadricauda & 1,9 & 47 & - \\
\hline Scenedesmus dimorphus & $16-40$ & $8-18$ & $21-52$ \\
\hline Spirulina maxima & $6-7$ & $60-71$ & $13-16$ \\
\hline Spirogyra sp. & $11-21$ & $6-20$ & $33-64$ \\
\hline Spirulina platensis & $4-9$ & $46-63$ & $8-14$ \\
\hline Synechococcus sp. & 11 & 63 & 15 \\
\hline Tetraselmis maculata & 3 & 52 & 15 \\
\hline
\end{tabular}

Fuente. Adaptada de Milano et al., 2016.

La especie Botryococcus braunii posee el mayor porcentaje de lípidos. Esta especie cuenta con un contenido de hidrocarburos que varía 
entre 15-80 \% (Jaimes y Kafarov, 2015). Según Levine y Fleurence (2018), los lípidos éter e hidrocarburos de cadena larga se acumulan en la matriz extracelular, lo cual se debe a la gran capacidad de esta especie de absorber $\mathrm{CO}_{2}$ en grandes cantidades, lo que acelera su crecimiento poblacional (Jaimes y Kafarov, 2015).

\subsection{Microalga oleaginosa}

Las microalgas oleaginosas requieren para su desarrollo factores ambientales tales como luz, $\mathrm{pH}$, salinidad, temperatura y concentración de nitrógeno. Este tipo de algas posen capacidad de almacenamiento de triglicéridos gracias a sus características fotobiosintéticas. Son capaces de fijar $\mathrm{CO}_{2}$ con el fin de producir diversas sustancias de valor industrial. De igual modo, frente a los cambios de condiciones ambientales responden con cambios al metabolismo de los lípidos. Así, por ejemplo, producciones de microalgas sin nitrógeno disponible resultan en mayores tasas de producción de lípidos por producción de mayor biomasa seca (Kiran, Pathak, Kumar y Deshmukh, 2016; Praveenkumar, Shameera, Mahalakshmi, Akbarsha y Thajuddin). Estos análisis se realizaron con tiempos de cultivo de seis días. 


\section{Métodos de cultivo de microalgas}

L

os métodos de cultivo toman en cuenta los siguientes parámetros requerimientos nutricionales, lumínicos y resistencia al estrés de la especie. De igual forma, las características del reactor en términos de superficie iluminada, transparencia y durabilidad del material, volumen - capacidad de escalado - y la orientación e inclinación que determinarán la velocidad de crecimiento de la microalga. También dependen del sistema de mezclado, de la dispersión de gases, los sistemas de limpieza y la regulación de la temperatura (Borowitzka, 1999).

\subsection{Sistemas abiertos}

Su efectividad se basa en factores como el bajo costo de mantenimiento para el cultivo de microalgas y su larga duración. Sin embargo, estos sistemas cuentan con desventajas tales como la baja accesibilidad de las células a la luz, la necesidad de tener grandes terrenos para los estanques y la exposición a las condiciones medioambientales que pueden llegar a alterar factores escenciales como, por ejemplo, el pH del agua.

Existen diferentes tipos de sistema de cultivo abierto en los cuales el agua y los nutrientes se suministran a las microalgas, de manera en que se canaliza y se reaprovecha agua de disposción industrial, vertimientos de agua cercanos o plantas de tratamiento de agua (Milano et al., 2016). El más común es el estanque de pista. En este, las microalgas, el agua y los nutrientes circulan por el estanque gracias a una rueda de paletas con el fin de mantener las microalgas a flote y facilitar la utlización del $\mathrm{CO}_{2}$ atmosférico. El estanque se fabrica con poca profundidad con el objetivo de maximizar la exposición de la luz de las microalgas para el proceso de la fotosíntesis. Sin embargo, el gran beneficio de este estanque es la posibilidad de reutilizar el $\mathrm{CO}_{2}$, el cual se burbujea a los desechos de 
nuevo dentro del estanque para ser reabsorbido por la siguiente cosecha de microalgas; es decir, se consigue un equilibrio de emisión y absorción (Milano et al., 2016).

Figura 2. Sistema de cultivo abierto

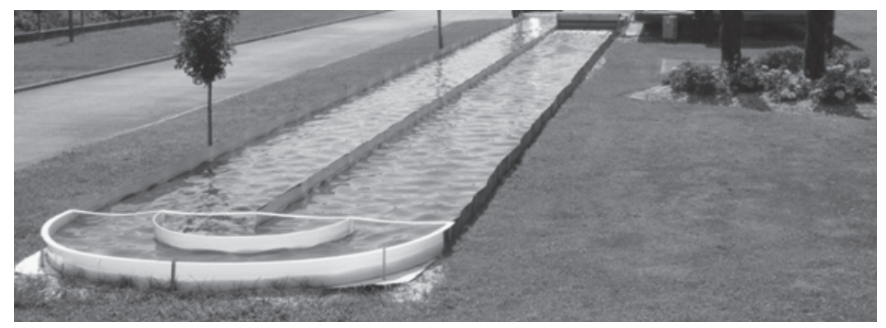

Fuente. Acualgae, s.f.

\subsection{Sistema cerrado}

Este sistema permite controlar los parámetros fundamentales para el crecimiento y la supervivencia de las microalgas. También disminuyen los problemas que se presentan en los sistemas abiertos, lo que ha ocasionado la preferencia de este sistema sobre estos. Con el sistema cerrado es posible realizar cultivos hiperconcentrados, es decir, cultivos con inóculos mayores a $1,5 \mathrm{~g} / \mathrm{L}$, y así obtener altas densidades celulares — ADC $>3 \mathrm{~g} / \mathrm{L}$ - o ultra alta densidad celular, esto es, UADC entre 15 y $80 \mathrm{~g} / \mathrm{L}$.

Este tipo de cultivos se llevan a cabo mediante reactores cerrados tubulares y son particularmente atractivos por la robustez del sistema y la reducción del riesgo de contaminación (Chisti, 1989). Frente al sistema abierto, el sistema cerrado cuenta con una mayor efectividad en términos de fijación del $\mathrm{CO}_{2}$ que requieren las microalgas, pues es posible obtener condiciones controladas durante el desarrollo de los cultivos, evita el intercambio de gases y contaminantes y permite conservarlas y protegerlas de fenómenos ambientales dañinos, con lo que se logran valores de densidad poblacional que aumentan el nivel y la rapidez de productividad. 
Los fotobiorreactores pueden ser verticales u horizontales. Esto varia dependiendo del espacio disponible o del propósito para el cual se utilicen. En estos reactores la luz no se filtra directamente sobre la superficie del cultivo, sino que debe traspasar las paredes del sistema.

En términos industriales, los sistemas cerrados se consideran y utilizan por encima de los sistemas abiertos debido la obtención de productos de alto valor añadido, la facilidad de tener control sobre las condiciones necesarias para la supervivencia y el crecimiento del cultivo y, por ende, un aumento en la produccion de biomasa proveniente de las microalgas.

Figura 3. Sistema de cultivo cerrado. Fotorreactor tubular de configuración horizontal

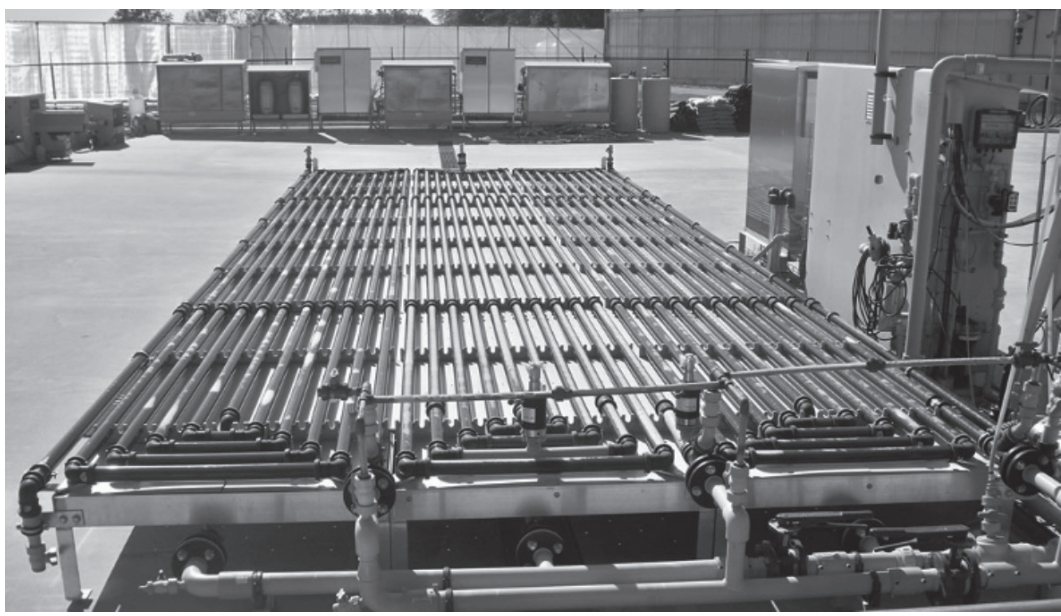

Fuente. Universidad Nacional de Colombia, 2017. 


\section{Sistemas de cosecha}

7 xiste una gran variedad de métodos para la cosecha de las microalgas. Esto varía conforme a la selección de la especie, la concentración de biomasa y el valor agregado del producto. Algunos de estos métodos son la centrifugación, la filtración, el ultrasonido y la electrofloculación.

\subsection{Centrifugación}

La centrifugación es un método de recolección que utiliza la fuerza centrífuga para separar mecánicamente las microalgas del agua con base en su diferencia de densidad (Figoli, Cassano y Basile, 2016). Este método es de los más eficientes con respecto a otras técnicas de recolección, por ser más rápido y extensible a todas las cepas de microalgas. En términos económicos, consume menor tiempo y es capaz de recuperar casi la totalidad de la biomasa que se encuentra libre de floculantes o productos químicos (Fuad et al., 2018). No obstante, a escala comercial no es práctico, ya que el aumento del costo del capital de inversión sumado al costo del consumo de energía de operación lo hace poco viable (Fuad et al., 2018).

\subsection{Floculación}

En algunos casos este método evita el uso de productos químicos adicionales y se considera un método de cosecha de bajo costo. La floculación se fundamenta en diferentes mecanismos tales como la neutralización de carga, el parche electrostático o mecanismos de barrido, dependiendo del agente floculante que se utilice. En términos generales, la adición de floculantes propicia la aglomeracion de las células de microalgas que originan los flóculos y aumentan de manera gradual su tamaño hasta sedimentar (Fuad et al., 2018). Sin agentes floculantes adicionados - floculantes químicos, naturales o cambios de $\mathrm{pH}$ - el tiempo de floculación del cultivo es más largo, de manera comparativa, debido al pequeño tamaño y a la carga superficial negativa de las microalgas, lo 
que contribuye a que el proceso sea más lento. Analizar estos aspectos es importante a fin de adoptar este método como una opción viable en la cosecha (Laamanen, Ross y Scott, 2016). Existe una gran variedad de factores que pueden afectar el proceso de floculación, entre los que se encuentran los tipos de floculantes, el $\mathrm{pH}$, la presencia de materia orgánica algal, la carga superficial, la biomasa inicial, la especie de microalga, etc. (Fuad et al., 2018).

La superficie de las células está cargada negativamente a causa de los grupos funcionales ionizados sobre la pared celular. La repulsión electrostática entre las células impide que se unan y adhieran de forma espontánea entre sí por fuerzas de Van der Waals. En estos procesos de floculación de microalgas se utiliza un agente floculante de tipo catiónico con el propósito de neutralizar la carga negativa de la superficie de las células, lo que facilita la formación espontánea de agregados de células o flóculos que sedimentan rápidamente (Chatsungnoen y Chisty, 2016). Algunas de las desventajas en este proceso es el alto riesgo de descomposición que sufren los productos, sumado al tiempo que requiere el proceso en comparación con otras alternativas de cosecha. Es por esto que se utilizan otros métodos más eficientes y seguros para los productos, aunque más costosos, como lo son la filtración, la separación ultrasónica, la separación magnética, la electrofloración o la centrifugación.

Recientemente, la floculación inducida por el aumento en el $\mathrm{pH}$ ha ganado atención para la cosecha de microalgas. La eficiencia de la floculación se incrementa a medida que el $\mathrm{pH}$ del medio alcanza niveles entre 11-12. El potencial zeta de las microalgas varía con diferente $\mathrm{pH}$, pero la carga superficial de estas es negativa a $\mathrm{pH}$ alcalino, con presencia de $\mathrm{OH}^{-}$ (Ummalyma, Mathew, Pandey y Sukumaran, 2016).

\subsection{Extracción de lípidos}

La extracción de lípidos de las microalgas se realiza de manera posterior a la extracción y purificación de la biomasa. El secado de la biomasa 
se realiza por exposición al sol, el método más económico en términos energéticos pero con un requerimiento de grandes superficies y tiempo (Milano et al., 2016).

Las microalgas contienen diferentes tipos de lípidos que se pueden usar en la producción de biocombustibles (Milano et al., 2016). En la extracción de los lípidos se utilizan métodos físicos, químicos y con fluidos supercríticos. La metodologia más usada en la producción de biocombustibles es la extraccion con solventes (Milano et al., 2016).

\section{Proceso de transesterificación}

$\mathbf{L}$

a reacción química que ocurre durante la producción de biodiesel en la fuente de lípidos se hacen reaccionar con un alcohol de bajo peso molecular para la obtención de ésteres metílicos o etílicos. Esta reacción se realiza en presencia de un catalizador - alcóxido- , cuya concentración es sumamente baja y no influye en la eficiencia de la esterificación. En este proceso se realiza un intercambio en el grupo éster: el glicerol por tres moléculas del nuevo alcohol de bajo peso molecular, tal como se muestra en la figura 4. Posterior a la reacción de transesterificación se realiza la separación del biodiesel y la glicerina por decantación.

Los productos de la reacción que se generan son los ésteres de los ácidos grasos con el alcohol de bajo peso molecular — biodiesel - y la glicerina. Una vez se obtienen estos productos se lleva a cabo el proceso de separación, neutralización y lavado. 
Figura 4. Reacción de transesterificación de un triglicérido con un alcohol para la obtención de alquil ésteres de ácidos grasos y glicerina<smiles></smiles>

Triglicerido

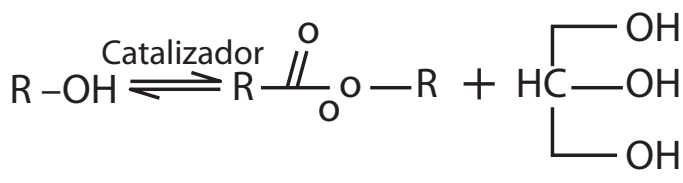

Éster

Glicerina

Fuente. Elaboración propia con software Chemsketch.

\section{Conclusiones}

$\mathrm{E}$ 1 uso continuo de combustibles derivados del petróleo es actualmente reconocido como insostenible debido a la disminución de las fuentes de petróleo y la contribución de estos combustibles a la acumulación de óxidos de azufre y productos de combustión incompleta en el medio ambiente. Por su parte, el biodiesel derivado de cultivos oleaginosos, de aceite de cocina usado y de grasa animal tienen una capacidad limitada para alcanzar objetivos de producción de biocombustibles, mitigación del cambio climático y crecimiento económico sin afectar la canasta familiar o aumentar la deforestación. Por el contrario, las microalgas se postulan como una fuente de biodiesel renovable capaz de satisfacer la demanda mundial de combustibles para el transporte sin poner en riesgo aspectos sociales ni ambientales. En suma, las microalgas, además de ser la materia prima para el biodiesel, brindan una alternativa en la descontaminación del ambiente, especialmente del aire y del agua. Su producción es viable, equitativa dentro de la sociedad y ambientalmente soportable, en una palabra, es sostenible. 


\section{Referencias}

Acualgae. (s. f.). Raceways. Recuperado de http://aqualgae.com/es/ productos-y-servicios/sistemas_cultivo_abiertos/

Amaro, H. M.; Guedes, A. C.; Malcata, F. X. (2011). Advances and perspectives in using microalgae to produce biodiesel. Applied Energy, 88(10), 3402-3410. DOI: https://doi.org/10.1016/j.apenergy.2010.12.014

Arunachalam, A. P.; Sivanandi, P.; Pandian, S.; Arumugamurthi, S. S.; Sircar, A. (2018). Optimization and kinetic studies on biodiesel production from microalgae (Euglena sanguinea) using calcium methoxide as catalyst. Energy sources, Part A: recovery, utilization, and environmental effects, 4l(12), 1497-1507. DOI: https://doi.org/10.1080/ 15567036.2018.1549124

Basha, S. A.; Gopal, K. R.; Jebaraj, S. (2009). A review on biodiesel production, combustion, emissions and performance. Renewable and Sustainable Energy Reviews, 13(6-7), 1628-1634. DOI: https://doi. org/10.1016/j.rser.2008.09.031

Becker, E. W. (2007). Micro-algae as a source of protein. Biotechnology Advances, 25(2), 207-210. DOI: https://doi.org/10.1016/j. biotechadv.2006.11.002

Bermeo Castillo, L. E. (2011). Estudio del cosechado de cultivos de microalgas en agua residual mediante técnicas de centrifugado (Tesis de maestría). Universidad de Cádiz. España.

Bohutskyi, P.; Liu, K.; Nasr, L. K.; Byers, N.; Rosenberg, J. N.; Oyler, G. A.; Betenbaugh, M.J.; Bouwer, E. J. (2015). Bioprospecting of microalgae for integrated biomass production and phytoremediation of unsterilized wastewater and anaerobic digestion centrate. Applied Microbiology and Biotechnology, 99(14), 6139-6154. DOI: https://doi. org/10.1007/s00253-015-6603-4 
Borowitzka, M. A. (1999). Commercial production of microalgae: ponds, tanks, and fermenters. Journal of Biotechnology. 70(1), 313-321. DOI: https://doi.org/10.1016/S0168-1656(99)00083-8

Chatsungnoen, T.; \& Chisti, Y. (2016). Harvesting microalgae by flocculation-sedimentation. Algal Research, 13, 271-283. DOI: https://doi. org/10.1016/j.algal.2015.12.009

Chen, C. Y.; Zhao, X. Q.; Yen, H. W.; Ho, S. H.; Cheng, C. L.; Lee, D. J.; Bai, F. W.; Chang, J. S. (2013). Microalgae-based carbohydrates for biofuel production. Biochemical Engineering Journal, 78, 1-10. DOI: https://doi.org/10.1016/j.bej.2013.03.006

Chisti, M. Y. (1989). Airlift bioreactors. Londres: Elsevier Applied Science.

Christenson, L.; Sims, R. (2011). Production and harvesting of microalgae for wastewater treatment, biofuels, and bioproducts. Biotechnology Advances, 29(6), 686-702. DOI: https://doi.org/10.1016/j. biotechadv.2011.05.015

Clebot, A. C. (2018). Bioprospección de cepas de microalgas de interés biotecnológico en el lixiviado proveniente del relleno. Encuentro de jóvenes investigadores. Universidad Nacional del Litoral. Recuperado de http://hdl.handle.net/11185/2066

Fuad, N.; Omar, R.; Kamarudin, S.; Harun, R.; Idris, A.; Wakg, W. A. (2018). Mass harvesting of marine microalgae using different techniques. Food and Bioproducts Processing, 112, 169-184. DOI: https:// doi.org/10.1016/j.fbp.2018.10.006

Figoli, A.; Cassano, A.; Basile, A. (Eds.). (2016). Membrane technologies for biorefining. Woodhead Publishing.

Gao, Y.; Skutsch, M.; Masera, O.; Pacheco, P. (2011). A global analysis of deforestation due to biofuel development (vol. 68). Cifor. 
Hernández-Pérez, A.; Labbé, J. I. (2014). Microalgas, cultivo y beneficios. Revista de Biología Marina y Oceanografía, 49(2), 157-173. DOI: https://doi.org/10.4067/S0718-19572014000200001

Jaimes, N. A.; Kafarov, V. (2015). Desarrollo preliminar de una metodología de suministro de $\mathrm{CO}_{2}$ a cultivos de Botryococcus Braunii para la producción de biocombustibles. Revista Ion, 28(2), 33-50. DOI: https:// doi.org/10.18273/revion.v28n2-2015003

Johnson, M. B.; Wen, Z. (2009). Production of biodiesel fuel from the microalga Schizochytrium limacinum by direct transesterification of algal biomass. Energy \& Fuels, 23(10), 5179-5183. DOI: https://oi. org/10.1021/ef900704h

Kiran, B.; Pathak, K.; Kumar, R.; Deshmukh, D. (2016). Growth pattern and biofuel production potential of newly isolated microalga, Chlorococcum sp. IM-03 under nitrogen limited conditions. Journal of Chemical Technology \& Biotechnology, 91(5), 1339-1344. DOI: https://doi. org/10.1002/jctb.4727

Laamanen, C. A., Ross, G. M.; Scott, J. A. (2016). Flotation harvesting of microalgae. Renewable and Sustainable Energy Reviews, 58, 75-86. DOI: https://doi.org/10.1016/j.rser.2015.12.293

Levine, I.; Fleurence, J. (Eds.). (2018). Microalgae in health and disease prevention. Academic Press. DOI: https://doi.org/10.1016/ B978-0-12-811405-6.00001-3

Ley 939 de 2004. (31 de diciembre de 2004). Por medio de la cual se subsanan los vicios de procedimiento en que incurrió en el trámite de la Ley 818 de 2003 y se estimula la producción y comercialización de biocombustibles de origen vegetal o animal para uso en motores diesel y se dictan otras disposiciones. Diario Oficial, núm. 45778. Congreso de Colombia. 
Loera-Quezada, M.; Olguín, E. J. (2010). Las microalgas oleaginosas como fuente de biodiesel: retos y oportunidades. Revista Latinoamericana de Biotecnología Ambiental y Algal, 1(1), 91-116.

López, J. (7 de marzo de 2018). Sector palmicultor: sinónimo de dinamismo y emprendimiento. Boletín El Palmicultor, (552-febrero), 14-16.

Mandal, S.; Mallick, N. (2009). Microalga Scenedesmus obliquus as a potential source for biodiesel production. Applied Microbiology and Biotechnology, 84(2), 281-291. DOI: https://doi.org/10.1007/ s00253-009-1935-6

Merchuk, J. C.; Gluz, M.; Mukmenev, I. (2000). Comparison of photobioreactors for cultivation of the red microalga Porphyridium sp. Journal of Chemical Technology \& Biotechnology: International Research in Process, Environmental \& Clean Technology, 75(12), 1119-1126. DOI: $\quad$ https://doi.org/10.1002/1097-4660(200012)75:12<1119::AIDJCTB329>3.0.CO;2-G

Milano, J.; Ong, H. C.; Masjuki, H. H.; Chong, W. T.; Lam, M. K.; Loh, P. K.; Vellayan, V. (2016). Microalgae biofuels as an alternative to fossil fuel for power generation. Renewable and Sustainable Energy Reviews, 58, 180-197. DOI: https://doi.org/10.1016/j.rser.2015.12.150

Monari, C.; Righi, S.; Olsen, S. I. (2016). Greenhouse gas emissions and energy balance of biodiesel production from microalgae cultivated in photobioreactors in Denmark: a life-cycle modeling. Journal of Cleaner Production, 112, 4084-4092. DOI: https://doi.org/10.1016/j. jclepro.2015.08.112

Praveenkumar, R.; Shameera, K.; Mahalakshmi, G.; Akbarsha, M. A.; Thajuddin, N. (2012). Influence of nutrient deprivations on lipid accumulation in a dominant indigenous microalga Chlorellasp., BUM11008: Evaluation for biodiesel production. Biomass and Bioenergy, 37, 6066. DOI: https://doi.org/10.1016/j.biombioe.2011.12.035 
Resolución 40174 de 2019. (24 de febrero de 2019). Por la cual se establece de forma temporal el porcentaje de mezcla de biocombustible para uso en motores diésel en algunos departamentos del país. Ministerio de Minas y Energía. Recuperado de: http:/ legal.legis.com.co/document/Index?obra $=$ legcol\&document $=1$ egc ol_f0d00442c6214b89a6b156987705e59c

Shuba, E. S.; Kifle, D. (2018). Microalgae to biofuels: 'Promising' alternative and renewable energy, review. Renewable and Sustainable Energy Reviews, 81, 743-755. DOI: https://doi.org/10.1016/j.rser.2017.08.042

Sreenivasan, B. (1978). Interesterification on fats. Journal of the American Oil Chemists'Society, 796-805. DOI: https://doi.org/10.1007/ BF02682651

Tripathi, R.; Gupta, A.; Thakur, I. S. (2019). An integrated approach for phycoremediation of wastewater and sustainable biodiesel production by green microalgae, Scenedesmus sp. ISTGA1. Renewable Energy, 135, 617-625. DOI: https://doi.org/10.1016/j.renene.2018.12.056

Universisdad Nacional de Colombia. (2017). Diseñan nuevo fotobiorreactor que mejoraría el cultivo de microalgas. Recuperado de https:// minas.medellin.unal.edu.co/noticias/facultad/1673-disenan-nuevofotobiorreactor-que-mejoraria-el-cultivo-de-microalgas

Ummalyma, S. B.; Mathew, A. K.; Pandey, A.; Sukumaran, R. K. (2016). Harvesting of microalgal biomass: efficient method for flocculation through $\mathrm{pH}$ modulation. Bioresource Technology, 213, 216-221. DOI: https://doi.org/10.1016/j.biortech.2016.03.114

Yeh, K. L.; Chang, J. S. (2011). Nitrogen starvation strategies and photobioreactor design for enhancing lipid content and lipid production of a newly isolated microalga Chlorella vulgaris ESP-31: implications for biofuels. Biotechnology Journal, 6(11), 1358-1366. DOI: https://doi. org/10.1002/biot.201000433 
Zhu, L.; Cheung, C. S.; Zhang, W. G.; Huang, Z. (2011). Combustion, performance and emission characteristics of a DI diesel engine fueled with ethanol-biodiesel blends. Fuel, 90(5), 1743-175. DOI: https://doi. org/10.1016/j.fuel.2011.01.024 